\title{
Accumulation of NACP/ $\alpha$-synuclein in Lewy body disease and multiple system atrophy
}

\author{
Mikio Shoji, Yasuo Harigaya, Atsushi Sasaki, Kenji Uéda, Koji Ishiguro, \\ Etsuro Matsubara, Mitsunori Watanabe, Masaki Ikeda, Mitsuyasu Kanai, \\ Yasushi Tomidokoro, Masami Shizuka, Masakuni Amari, Kenji Kosaka, Yoichi Nakazato, \\ Koichi Okamoto, Shunsaku Hirai
}

Abstract

Objectives-NACP/ $-\mathrm{-}$-synuclein is an aetiological gene product in familial Parkinson's disease. To clarify the pathological role of NACP/ $\alpha$-synuclein in sporadic Parkinson's disease and other related disorders including diffuse Lewy body disease (DLBD) and multiple system atrophy (MSA), paraffin sections were examined immunocytochemically using anti-NACP/ $\alpha$-synuclein antibodies.

Methods-A total of 58 necropsied brains, from seven patients with Parkinson's disease, five with DLBD, six with MSA, 12 with Alzheimer's disease, one with Down's syndrome, one with amyotrophic lateral sclerosis (ALS), three with ALS and dementia, one with Huntington's disease, two with progressive supranuclear palsy (PSP), one with Pick's disease, one with myotonic dystrophy, and three with late cerebellar cortical atrophy (LCCA), and 15 elderly normal controls were examined.

Results-In addition to immunoreactive Lewy bodies, widespread accumulation of $\mathrm{NACP} / \alpha$-synuclein was found in neurons and astrocytes from the brainstem and basal ganglia to the cerebral cortices in Parkinson's disease/DLBD. NACP/ $\alpha-$ synuclein accumulates in oligodendrocytes from the spinal cord, the brain stem to the cerebellar white matter, and inferior olivary neurons in MSA. These widespread accumulations were not seen in other types of dementia or spinocerebellar ataxia.

Conclusion-Completely different types of NACP/ $\alpha$-synuclein accumulation in Parkinson's disease/DLBD and MSA suggest that accumulation is a major step in the pathological cascade of both diseases and provides novel strategies for the development of therapies.

(F Neurol Neurosurg Psychiatry 2000;68:605-608)

Keywords: Parkinson's disease; multiple system atrophy; NACP; $\alpha$-synuclein

Dr Mikio Shoji, Department

of Neurology, Gunma

University School of

Medicine, 3-39-22

Showamachi, Maebashi,

Gunma 371-8511, Japan

emailmshoji@

akagi.sb.gunma-u.ac.jp

Received 31 March 1999 and in final form

24 November 1999

Accepted 3 December 1999 are the most constant pathological findings in Parkinson's disease. ${ }^{23}$ Although treatment with levodopa or dopamine receptor agonists based on the depletion of striatal dopamine markedly improves parkinsonism, patients with Parkinson's disease still become disabled within 5 to 10 years with the advancement of disease. A non-A $\beta$ component of Alzheimer's disease amyloid (NAC) was identified as the second intrinsic component in amyloid from Alzheimer's disease brains. ${ }^{4}$ The cloned NAC precursor protein termed $\mathrm{NACP}^{4}$ (also called $\alpha$-synuclein ${ }^{5}$ ), a presynaptic nerve terminal protein, ${ }^{6}$ has been identified as the causal gene product in five families with Parkinson's disease (one Italian ${ }^{7}$ and three Greek families ${ }^{7}$ and one German family ${ }^{8}$ ). To clarify the pathological role of NACP in sporadic Parkinson's disease and other related disorders including diffuse Lewy body disease (DLBD) ${ }^{910}$ and multiple system atrophy (MSA), ${ }^{11}$ paraffin sections were examined immunocytochemically in the present study.

Materials and methods

A total of 58 necropsied brains from seven patients with Parkinson's disease (age 64-81), five with DLBD (age 73-84), six with MSA (age 51-66), 12 with Alzheimer's disease (age 48-95), one with Down's syndrome, one with amyotrophic lateral sclerosis (ALS), three with ALS and dementia, one with Huntington's disease, two with progressive supranuclear palsy (PSP), one with Pick's disease, one with myotonic dystrophy, three with late cerebellar cortical atrophy (LCCA), and 15 elderly normal controls (age 45-91) were examined. The diagnosis of DLBD was based on the clinical criteria of the DLB international workshop for dementia with Lewy bodies ${ }^{12}$ and the pathological appearance of many cortical Lewy bodies detected using haematoxylin and eosin staining.

The anti-NACP antibodies: $\mathrm{MDV}^{13}$ - a polyclonal antibody against a synthetic 15 amino acid peptide of the N-terminus of NACP; NACPN - a polyclonal antibody against a synthetic 30 amino acid peptide of the $\mathrm{N}$-terminus of NACP; NACPC-a polyclonal antibody against a synthetic 30 amino acid peptide of the C-terminus of NACP, and anti-NAC domain antibody (EQV $)^{13}$ - a polyclonal antibody against 15 amino acid peptides of the N-terminus of NAC - were used at a dilution of $1: 1000$. Paraffin sections were pretreated with $99 \%$ formic acid for 3 minutes, incubated 

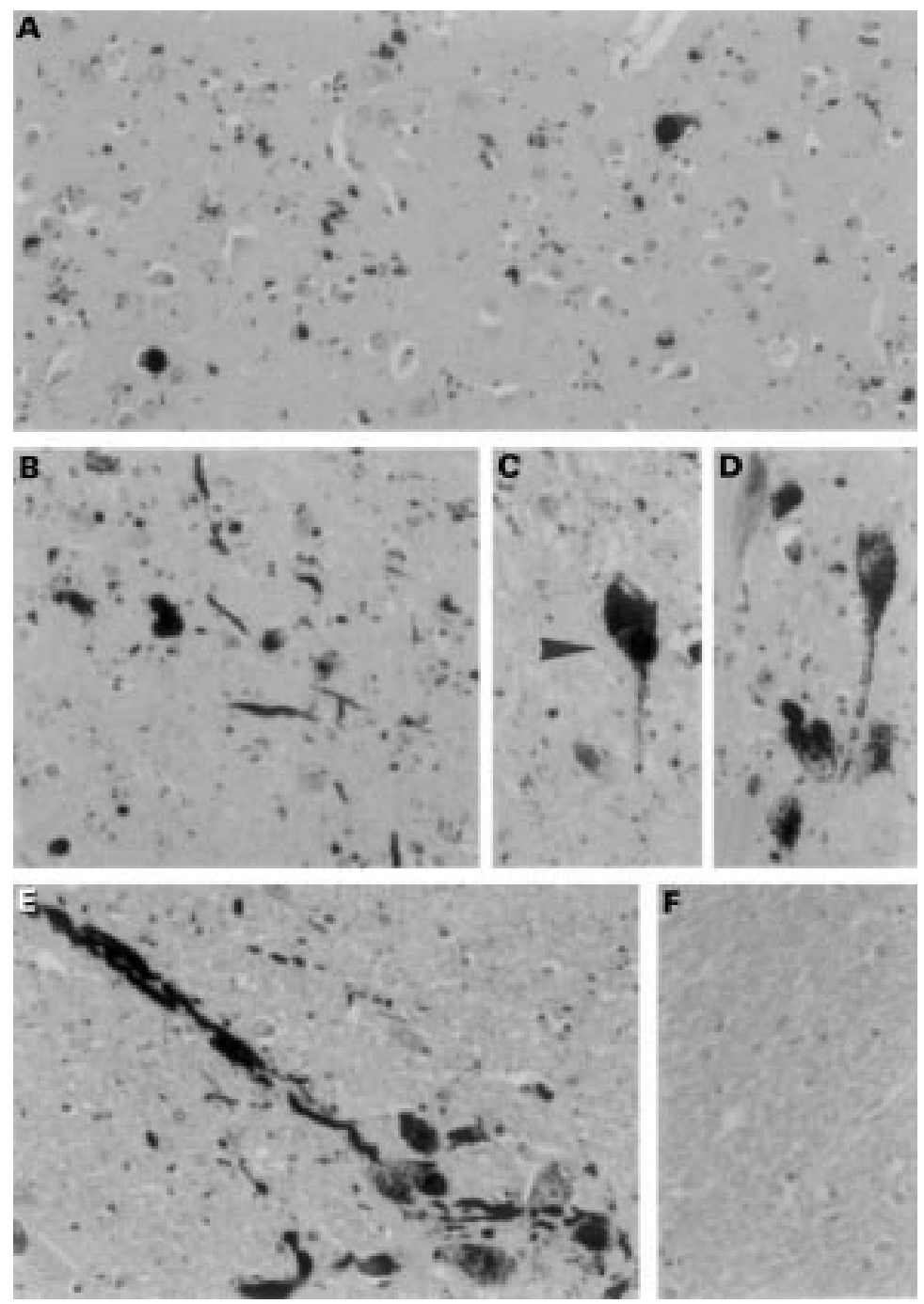

Figure 1 Immunostaining of $N A C P / \alpha$-synuclein in a parkinsonian brain using $M D V$ antibody (1:1000). A few cortical Lewy bodies, dystrophic neurites, and astrocytes are found in the cerebral cortex $(A \times 160)$, and basal ganglia $(B \times 160)$. In the substantia nigra, typical Lewy bodies ( $C$ arrow head $\times 320$ ), granular intraneural NACP accumulation $(C \times 320)$, perineural NACP accumulation $(D \times 320)$, and giant swelling of neuronal processes are detected (E×160). No immunoreactivity is seen in the crus cerebri in the midbrain $(F \times 160)$.

with primary antibodies for 3 hours, and then immunostained using an ABC immunostaining kit (Vector, Burlingame, CA, USA). Haematoxylin was used for nuclear staining. These preimmune sera did not show any immunoreactivity in any sections.

\section{Results}

In the Parkinson's disease brains, all antibodies-MDV, NACPN, NACPC, and EQV - labelled many Lewy bodies in the substantia nigra and locus ceruleus, as reported
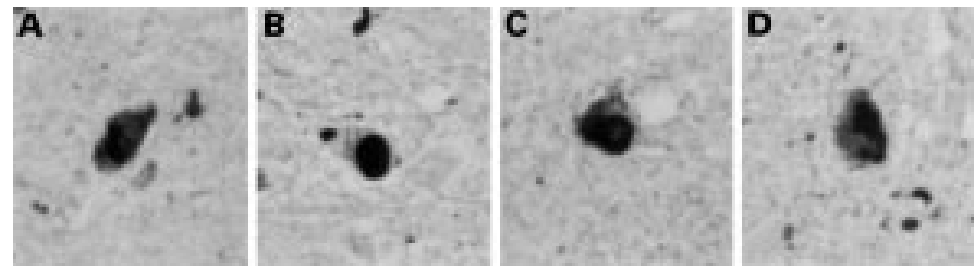

Figure 2 Immunostaining of Lewy body in the midbrain of a patient with Parkinson's disease using $M D V, N A C P N, E Q V, N A C P C$ antibodies. (A) MDV×480; (B) $N A C P N \times 480$; (C) EQV $\times 480$; (D) $N A C P C \times 480$. previously $^{14-16}$ (fig $1 \mathrm{C}$ arrow head). In addition, many small granules in neurons (fig $1 \mathrm{C}$ and D), a giant swelling of neuronal processes, and many dystrophic neurites (fig 1 E) were detected in the grey matter from the pons and midbrain to the basal ganglia and cerebral cortex. Extensive dystrophic neurites and intraneural granules in basal ganglia (fig $1 \mathrm{~B}$ ), a moderate number of dystrophic neurites, and a few cortical-type Lewy bodies in the cerebral cortices (fig 1 A) were recognised. Another interesting finding was the presence of immunoreactive astrocytes in Parkinson's disease brains (fig $1 \mathrm{~A}$ ). The severity of the immunoreactive astrocytosis was correlated with the appearance of cortical Lewy bodies and dystrophic neurites. These immunoreactivities were not detected in cerebral white matter, cerebellar white or grey matter, crus cerebri (fig $1 \mathrm{~F}$ ), or the white and grey matter of the lower brainstem and spinal cord. All antibodiesMDV, NACPN, EQV, and NACPC-showed similar immunoreactivities (fig 2).

Diffuse Lewy body disease is the third major cause of dementia after Alzheimer's disease and vascular dementia, with symptoms of parkinsonism and dementia. ${ }^{9} 10$ All antibodiesMDV, NACPN, EQV, and NACPC-labelled many immunoreactive cortical Lewy bodies in the fifth and sixth layers, and dystrophic neurites and prominent astrocytosis in all layers of the cerebral cortex in all cases of DLBD (fig 3 $\mathrm{A}$ and B). Although many Lewy bodies, dystrophic neurites, and astrocytes were also recognised in the basal ganglia (fig $3 \mathrm{C}$ ), NACP accumulation in the DLBD brainstem was at a similar level as that in the Parkinson's disease brains (fig $3 \mathrm{E}$ ). However, it was not found in the cerebellum (fig $3 \mathrm{D}$ ) or lower brainstem.

Marked accumulation of NACP was not seen in the brains of patients with the other kinds of dementia examined here; two cases of progressive supranuclear palsy, one case of Huntington's disease, one case of Pick's disease, three cases of amyotrophic lateral sclerosis (ALS) with dementia, and one case of myotonic dystrophy. One ALS brain and all of the 15 elderly normal control brains did not show NACP accumulation. We subsequently examined 12 brains from sporadic Alzheimer's disease and a Down's syndrome brain. Although the frozen sections of these brains showed weak immunoreactive senile plaques using the EQV antibody alone, no paraffin sections from the 12 Alzheimer's disease brains or the Down's syndrome brain showed any significant NACP immunoreactivity in neurons, astrocytes, or senile plaques.

Multiple systems atrophy shows varying degrees of parkinsonism, automatic dysfunction, and cerebellar ataxia. ${ }^{11}$ Recent studies have shown the presence of NACP in glial cytoplasmic inclusions (GCI) in the brainstem and cerebellar white matter. ${ }^{17-20}$ All MDV, NACPN, EQV, and NACPC antibodies showed extensive distributions of NACPimmunoreactive oligodendrocytes in the cerebellar white matter (fig 4 B), basal ganglia (fig $4 \mathrm{C}$ ), brainstem, and spinal cord of the MSA brains. Large accumulations of NACP in 
oligodendrocytes were seen in the internal capsule, lateral and medial medullary laminae, putamen, and globus pallidus, crus cerebri (fig 4 D), cerebellar white matter, cerebellar peduncles, pontine basis (fig $4 \mathrm{E}$ ), medulla oblongata (fig $4 \mathrm{H}$ ), and from the cervical to lumbar spinal cord. These oligodendrocytes were rare in the cerebral grey and white matter (fig $4 \mathrm{~A}$ ), cerebellar cortex and dentate nuclei, the dorsal part of the brainstem, and the posterior funiculus of the spinal cord. There were accumulations of NACP in neurons and processes in the inferior olivary nucleus alone (fig 4 G). Degenerated cerebellar Purkinje cells and the midbrain pigmented neurons (fig $4 \mathrm{H}$ ) were not labelled by any of the antibodies. Such accumulations were not seen in LCCA brains.
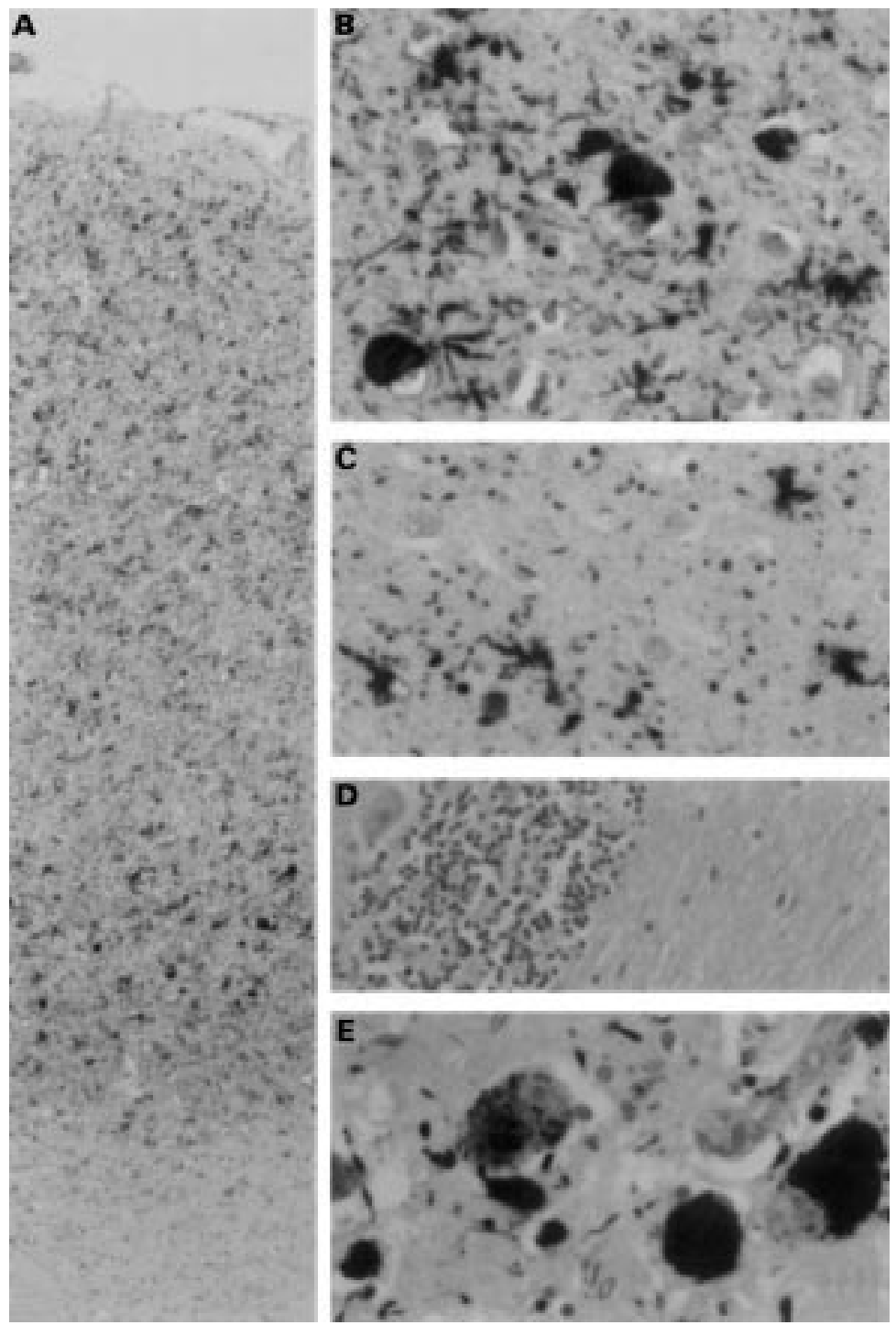

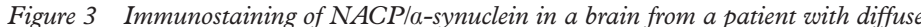
Lewy body disease $(D L B D)$ using $M D V$ antibody (1:1000). $(A \times 32, B \times 320)$ Extensive immunoreactivity in many cortical Lewy bodies, dystrophic neurites, and astrocytes is seen in the cerebral cortex. (C×320) These immunoreactive cortical Lewy bodies, dystrophic neurites, and astrocytes are also seen in the basal ganglia. $(D \times 160)$ The cerebellar cortex and white matter are not stained. (E×320) In the substantia nigra, extensive accumulation

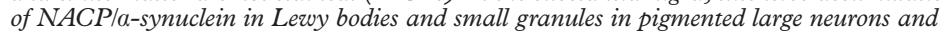
many dystrophic neurites are labelled.

\section{Discussion}

The findings from Parkinson's disease brains indicate that molecules of NACP accumulate as small granules in neurons, and as diffuse deposits in neuronal processes, in addition to Lewy bodies. These intracellular accumulations of NACP were distributed more extensively in larger areas than those detected using haematoxylin and eosin staining, which showed only the presence of Lewy bodies and neuronal cell loss in the substantia nigra and other brainstem nuclei, and in intact basal ganglia and cortical cortices in Parkinson's disease brains. Although NACP is considered to be a synaptic protein, NACP was detected in cortical astrocytes. It accumulates not as a mere component of Lewy bodies, but as independent deposits with abnormal structures in neural/astrocytic cell systems more extensively than those detected using conventional pathological examination. These newly detected widespread accumulations of NACP may disturb connections between the brainstem and basal ganglia, and among nuclei in the basal ganglia, and may be another origin of clinical parkinsonism, as seen in secondary Parkinson's syndrome.

The distribution and the cell type of NACP accumulation were essentially the same in the Parkinson's disease and DLBD brains. The only difference was the appearance of more Lewy bodies, dystrophic neurites, and astrocytes in the basal ganglia and the cortex of DLBD brains. These findings suggest that DLBD is essentially identical to Parkinson's disease in terms of NACP accumulation. The diffuse damage to cerebral cortices by severe astrocytosis, Lewy bodies, and dystrophic neurites may cause clinical dementia in DLBD.

The paraffin embedding may have caused the loss of the epitope of the NAC domain in senile plaques, as previously demonstrated. ${ }^{21}$ These findings suggested that the newly detected intracellular accumulations of NACP are a specific event in Lewy body disease (Parkinson's disease and DLBD) by contrast with Alzheimer's disease, where extracellular accumulations of degradation fragments of NACP, such as NAC, occur with $A \beta$ amyloid deposits. As reactive astrocytes in the brains with other diseases were not labelled by NACP antibodies, the appearance of immunoreactive NACP astrocytosis is considered to be specific to Lewy body disease.

NACP accumulates widely and specifically in oligodendroglia and olivary neurons in MSA. These completely different types of accumulation of NACP can easily identify MSA as distinct from Lewy body disease and other neurodegenerative diseases. The conduction disturbances in the subcortical white matter of the basal ganglia, cerebellum, and brainstem due to oligodendroglial damage may be correlated with the complex symptoms of MSA.

As the degraded fragment of NACP, NAC, is a component of extracellular Alzheimer's amyloids, NACP is a key step in the pathological cascades of major neurodegenerative diseases. The binding of NAC to $A \beta$ is considered to be 

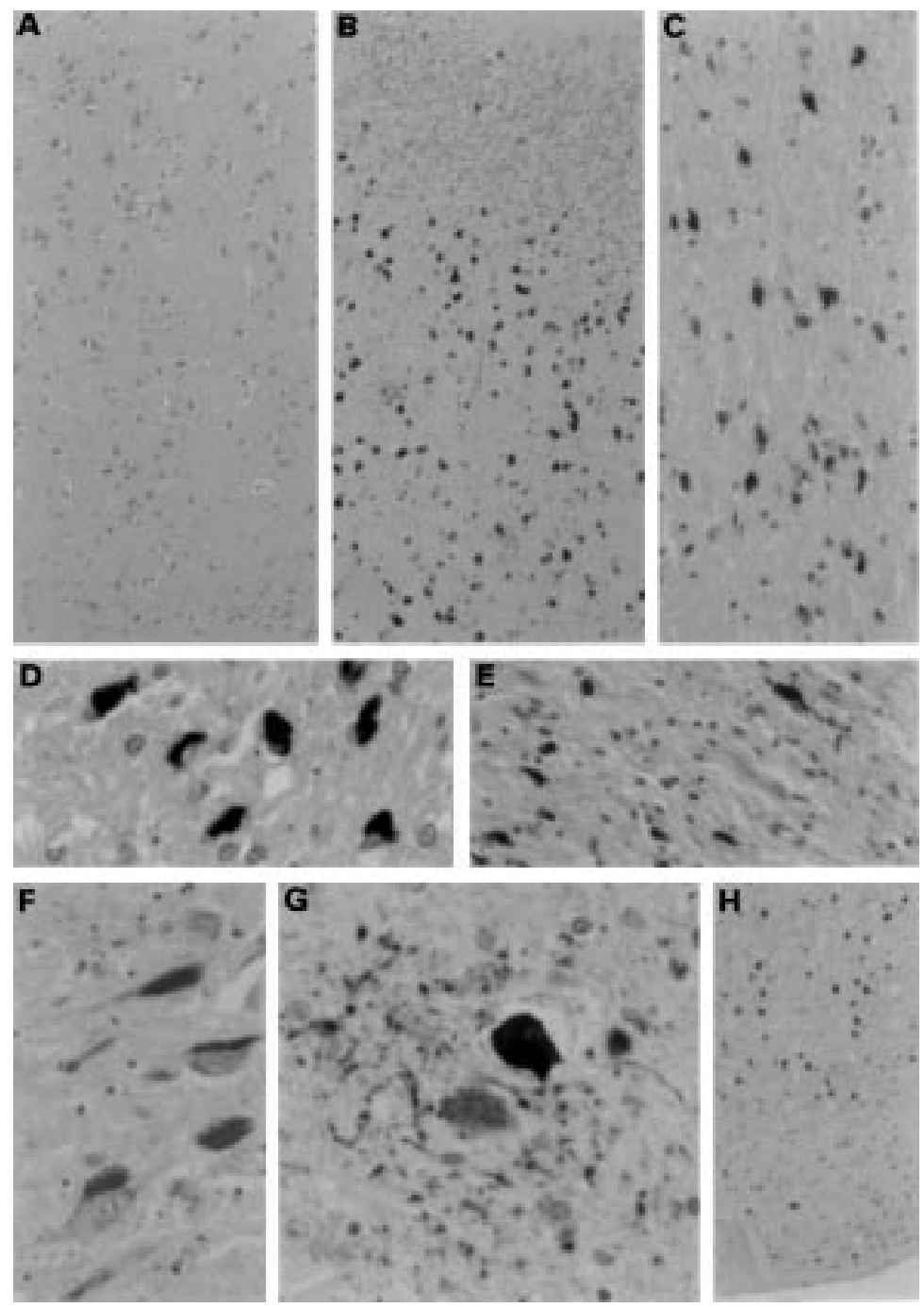

Figure 4 Immunostaining of $N A C P / a-$ synuclein in a brain with multiple system atrophy (MSA) by MDV antibody (1:1000). (A×64) Although no immunoreactivity is seen in the grey and white matter of the cerebral cortex, $(B \times 64)$ many oligodendrocytes are extensively labelled in the cerebellar white matter. $(C \times 160)$ These immunoreactive oligodendrocytes are seen in the basal ganglia, $(D \times 320)$ crus cerebri, and $(E \times 160)$ potine basis. $(F \times 320)$ The midbrain pigmented neurons are not stained. (G×320) NACP/a-synuclein accumulated only in neurons and neurites of the inferior olivary nucleus. (H×64) Many immunoreactive oligodendrocytes are seen in the pyramid in the medulla oblongata.

the pathological origin of amyloid formation. ${ }^{22}$ The accumulations of NACP detected in the present study were not stained by Congo red, suggesting that they may not have a significant level of the $\beta$-pleated sheet configuration, as seen in senile plaque amyloid (data not shown). Mutant NACP genes of familial Parkinson's disease are the origins of the pathological cascades. Other upstream factors in the cascades, such as disturbance of axonal transport, proteolytic degradations, and the alternative synthesis of NACP in different cell lines, remain to be clarified. The present findings support the concept that NACP accumulation shares pathological similarities with other neurodegenerative diseases, many of which have recently been shown to involve accumulations of aetiological gene products in the brains. ${ }^{23}$ The study of tau has contributed to clarification of the pathogenesis of many neurodegenerative diseases. Studies on NACP accumulation may provide an understanding of Lewy body disease and MSA and clarify novel strategies for the development of therapies leading to a cure for Lewy body disease and MSA.

This work was supported by the Life Science Foundation, the Sasakawa Health Science Foundation, Uehara Memorial Foundation, the Primary Amyloidosis Research Committee of the Ministry and Welfare of Japan, and the Ministry of Education, Science, and Culture of Japan.

1 Parkinson J. An essay on the shaking palsy. In: Critcheley M, eds. Fames Parkinson. London: Macmillan, 1955.

2 Levy FH. Paralysis agitans 1. Pathologisch Anatomie. In: Lewandowski M, eds. Handbuch der Neurologie III. Berlin Springer Verlag 1912:920-33.

3 Trétiakoff MC. Contribution a l'étude de l'anatomie pathologique de Locus Niger de Soemmerling [thesis]. Paris: Université de Paris, 1919.

4 Uéda K, Fukushima H, Masliah E, et al. Molecular cloning of cDNA encoding an unrecognized component of amyloid in Alzheimer disease. Proc Natl Acad Sci U S A 1993;90:11282-6.

5 Jakes R, Spillantini MG, Goedert M. Identification of two distinct synucleins from human brain. FEBS Lett 1994; 345:27-32.

6 Iwai A, Masliah E, Yoshimoto M, et al. The precursor protein of non-A $\beta$ component of Alzheimer's disease amyloid is a presynaptic protein of the central nervous system. Neuis a presynaptic protein
ron 1995;14:467-75.

7 Polymeropoulos MH, Lavedan C, Leroy E, et al. Mutation in the $\alpha$-synuclein gene identified in families with Parkinson's disease. Science 1997;276:2045-7.

8 Krüger R, Kuhn W, Muller T, et al. Ala30Pro mutation in the gene encoding $\alpha$-synuclein in Parkinson's disease. Nat Genet 1998;18:106-8.

9 Yoshimura $M$. Cortical changes in the parkinsonian brain: a contribution to the delineation of "diffuse Lewy body disease". F Neurol 1983;229:17-32.

10 Kosaka K, Yoshimura M, Ikeda K, et al. Diffuse type of Lewy body disease: progressive dementia with abundant cortical Lewy bodies and senile changes of varying degree. A new disease? Clin Neuropathol 1984;3:185-92.

11 Graham J, Oppenheimer D. Orthostatic hypotension and nicotine sensitivity in a case of multiple system atrophy. $\mathcal{F}$

12 McKeith IG, Galasko D, Kosaka K, et al. Consensus guidelines for the clinical and pathologic diagnosis of dementia with Lewy bodies (DLB): report of the consortium on DLB international workshop. Neurology 1996;47:1113-24.

13 Ishimaru $\mathrm{H}$, Ueda $\mathrm{K}$, Takahashi A, et al. Changes in presynaptic protein NACP/ $\alpha$-synuclein in an ischemic gerbil hippocampus. Brain Res 1998;788:311-4.

14 Spillantini MG, Schmidt ML, Lee VM, et al. $\alpha$-synuclein in Lewy bodies. Nature 1997;388:839-40.

15 Baba M, Nakajo S, Tu PH, et al. Aggregation of $\alpha$-synuclein in Lewy bodies of sporadic Parkinson's disease and dementia with Lewy bodies. Am F Pathol 1998;152:879-84.

16 Takeda A, Mallory M, Sundsmo M, et al. Abnormal accumulation of $\mathrm{NACP} / \alpha$-synuclein in neurodegenerative disorders. Am f Pathol 1998;152:367-72.

17 Nakazato Y, Yamazaki H, Hirato J, et al. Oligodendroglial microtubular tangles in olivopontocerebellar atrophy. $\mathcal{f}$ Neuropathol Exp Neurol 1990;49:521-30.

18 Papp MI, Lantos PL. Accumulation of tubular structures in oligodendroglial and neuronal cells as the basic alteration in multiple system atrophy. F Neurol Sci 1992;107:172-82.

19 Mezey E, Dehejia A, Harta G, et al. $\alpha$ Synuclein in neurodegenerative disorders: murderer or accomplice? Nat Med 1998;4:755-7

20 Tu PH, Galvin JE, Baba M, et al. Glial cytoplasmic inclusions in white matter oligodendrocytes of multiple system atrophy brains contain insoluble $\alpha$-synuclein. Ann Neurol 1998;44:415-22

21 Masliah E, Iwai A, Mallory M, et al. Altered presynaptic protein NACP is associated with plaque formation and neurodegeneration in Alzheimer's disease. Am $\mathcal{f}$ Pathol 1996;148:201-10.

22 Yoshimoto $M$, Iwai A, Kang D, et al. NACP, the precursor protein of the non-amyloid $\beta / A 4$ protein (A $\beta$ ) component of Alzheimer disease amyloid, binds $A \beta$ and stimulates $A \beta$ aggregation. Proc Natl Acad Sci U S A 1995;92:9141-5.

23 Hardy J, Gwinn-Hardy K. Genetic classification of primary neurodegenerative disease. Science 1998;282:1075-9. 